\title{
SKCG-I: a new candidate growth regulatory gene at chromosome I lq23.2 in human sporadic Wilms tumours
}

\author{
KP Singh ${ }^{*, 1}$ and D Roy ${ }^{2}$ \\ 'Department of Biology, Texas Southern University, Houston, TX 77004, USA; ${ }^{2}$ Department of Environmental and Occupational Health, Stempel School \\ of Public Health, Florida International University, Miami, FL 33199, USA
}

\begin{abstract}
Using arbitrary primed-PCR (AP-PCR), we have identified a novel genetic alteration located at chromosome I I q23.2 and this genetic alteration was common in $38 \%$ of the human Wilms tumour samples analysed. Further characterisation by cloning and sequencing of this genomic region revealed that it represents a part of an uncharacterised gene. We have named this gene as Sporadic Kidney Cancer Gene-I (SKCG-I). Using fluorescence in situ hybridisation (FISH) approach, we established its localisation on the chromosome I I q23.2. Northern analysis revealed the transcript size of SKCG-I of $2.09 \mathrm{~kb}$ and this was further confirmed by full-length cDNA sequence. Sequence analysis revealed an active translation start site (ATG sequence), a polyadenylation signal sequence (AATAAA), and an open reading frame (ORF) encoding a peptide of 124 amino acids in the CDNA sequence of SKCG-I. Analysis of genomic sequence of SKCG-I revealed a promoter region containing TATA box located at - I 3 bp upstream of transcription start site. The AP-PCR, SCAR, and Southern blot analyses indicated genomic loss of SKCG-I in Wilms tumours. The transcript of SKCG-I was abundantly present in brain, kidney, liver, testis, salivary gland, foetal brain, foetal liver, whereas relatively lower expression in heart, stomach, prostate and no expression in spleen, colon, lung, small intestine, muscle, adrenal gland, uterus, skin, PBL, and bone marrow was detected. The expression of this gene transcript was either very less or undetectable in Wilms and breast tumours compared to their matched uninvolved tissues. Inhibition of SKCG-I by siRNA resulted in increased cell proliferation of kidney epithelial cells. Based on the presence of two transmembrane regions in its peptide, SKCG-I has been predicted as a transmembrane protein. Thus, the findings of this study revealed (i) SKCG-I, a new gene located at I I q23.2 and harbouring genetic alteration in Wilms tumours, (ii) the presence of SKCG-I gene transcripts in various human normal tissues and its lower expression or absence in Wilms and breast tumours indicate that it may be associated with tumour growth suppressor activity, (iii) the presence of an open reading frame in the cDNA sequence of SKCG-I indicates that it has potential to encode a protein, (iv) increased cell growth by silencing this gene in HEK293 cells further supports a potential role of this gene in growth of kidney epithelial cells. Our findings suggest that SKCG-I may have a tumour suppressor role, and implicate genetic alteration in this gene as a potential oncogenic pathway and therapeutic target in kidney and breast cancer.
\end{abstract}

British Journal of Cancer (2006) 94, I524- 1532. doi: I0.1038/sj.bjc.6603090 www.bjcancer.com

Published online II April 2006

(c) 2006 Cancer Research UK

Keywords: novel gene; Wilms tumour; growth regulatory gene; chromosome I lq23.2

\begin{abstract}
Altered expression of those genes that control cell proliferation and differentiation is a common end result of genetic alterations that occur in human tumours and is a crucial step in tumorigenesis. Identification and characterisation of all possible genetic alterations that predispose to cancer are of utmost importance to understand the development of cancer. In this study, by using arbitrary primed-PCR (AP-PCR) assay, we identified a new gene, SKCG-1, harbouring genetic alteration in $38 \%$ of the human sporadic Wilms tumour samples analysed. Wilms tumour or Nephroblastoma is an embryonal malignancy of the kidney,
\end{abstract}

\footnotetext{
*Correspondence: Dr KP Singh;

E-mail: Kamaleshwar9@aol.com

NCBI GenBank accession number for SKCG-1 is AY662656.

Received 22 September 2005; revised 6 February 2006; accepted 14 March 2006; published online II April 2006
}

affecting about 1 in 10000 children (Matsunaga, 1981). It comprises about $6 \%$ of all childhood cancers and is a major paediatric cancer burden at the global level (Volkher et al, 2001). The important discoveries made in the past several years regarding molecular events underlying Wilms tumorigenesis have indicated the involvement of two tumour suppressor genes namely WT1 and WT2 located at $11 \mathrm{p} 13$ and $11 \mathrm{p} 15$, respectively (Mannens et al, 1988; Koufos et al, 1989; Call et al, 1990; Gessler et al, 1990). Besides these two genes, many other mutational events, such as allelic loss of chromosome 16q (Maw et al, 1992), loss of heterozygosity at 11q23.3-q-ter region (Radice et al, 1995), and high rate of P53 mutations located at 17p13 (Bardeesy et al, 1994) in Wilms tumour indicate the involvement of these genes/loci with Wilms tumour formation. Association of a particular genetic abnormality with specific histopathology of Wilms tumour indicates that WT development involves several, probably alternative, genetic pathways, but there is still an incomplete picture as 
to the identity of most of these genes, or the mechanisms by which they are controlled (Grundy et al, 1994; Hing et al, 2001). Thus, a complete description of molecular defects in individual WTs is highly warranted.

Using AP-PCR method, we have recently identified a novel mutation located at $17 \mathrm{q} 11.2$ in $80 \%$ of the sporadic breast tumour samples analysed (Singh and Roy, 2001). Here we report the identification and characterisation of a new gene, SKCG-1, which is altered in human Wilms and breast tumours. We have cloned the full-length cDNA sequence of SKCG-1, established its chromosomal localisation, determined its structural analysis and coding potential, analysed its expression in various normal human tissues, including Wilms and breast tumours and their corresponding uninvolved tissues, and tested its function in cell growth by silencing it.

\section{MATERIALS AND METHODS}

\section{Chemicals}

The AmpliTaq DNA polymerase (Stoffel fragment), dNTPs and mineral oil were purchased from PE Applied Biosystems (Foster City, CA, USA). A set of 20 OPA series oligonucleotide random ten-mer primers was purchased from Operon technologies (Alameda, CA, USA). The DIG high prime DNA labelling and detection kit was obtained from Roche Diagnostics (Indianapolis, IN, USA).

\section{DNA and RNA extraction}

Thirteen human Wilms tumour tissues (six Caucasian American cases, six African-American cases, and one Asian), out of which 10 with matching tumour-normal pair and three tumours without matching normal as well as seven breast tumours with matching normal tissues were obtained through NCI Tissue Procurement Facility, Comprehensive Cancer Center (University of Alabama at Birmingham, USA). Human embryonic kidney epithelial cell line, HEK-293, was obtained from ATCC and propagated in DMEM/F12 growth medium with $10 \%$ FBS. Genomic DNAs were isolated from tumour and corresponding normal frozen tissues following the methods as described previously by us (Singh and Roy, 1999). Total RNA was isolated from the kidney and breast tumours, their corresponding uninvolved tissues, as well as from in vitro grown embryonic kidney epithelial cells (HEK-293) following the Trizol method (Invitrogen, Carlsbad, CA, USA). The DNA and RNA were quantified spectrophotometrically, and purity as well as integrity was checked by ethidium bromide staining after resolving on $1.5 \%$ agarose gel (for DNA) and on $1 \%$ agarose gel in $1 \times$ formaldehyde buffer (for RNA).

\section{AP-PCR}

AP-PCR was performed following the method as described previously by us (Singh and Roy, 2001). Amplifications were carried out in $25 \mu \mathrm{l}$ of reaction mixture containing $100 \mu \mathrm{M}$ each of dATP, dCTP, dGTP, dTTP; $2.5 \mathrm{mM} \mathrm{MgCl}_{2} ; 2.5 \mu \mathrm{l}$ of $10 \times$ enzyme assay buffer, 1.5 unit of Taq DNA polymerase (stoffel) and $75 \mathrm{ng}$ of genomic DNA. From a total of 15 primers used, the OPA14 $\left(5^{\prime}\right.$-TCTGTGCTGG- $\left.3^{\prime}\right)$ was the only primer that revealed genetic alteration in the AP-PCR profile of Wilms tumour DNA. DNA amplification was performed in a Perkin-Elmer Cetus DNA thermal cycler programmed for 45 cycles: first cycle $(3.5 \mathrm{~min}$ at $92^{\circ} \mathrm{C}, 1 \mathrm{~min}$ at $34^{\circ} \mathrm{C}, 2 \mathrm{~min}$ at $72^{\circ} \mathrm{C}$ ), next 44 cycles $\left(1 \mathrm{~min}\right.$ at $92^{\circ} \mathrm{C}$, $1 \mathrm{~min}$ at $34^{\circ} \mathrm{C}, 2 \mathrm{~min}$ at $72^{\circ} \mathrm{C}$ ), followed by a final extension cycle of $15 \mathrm{~min}$ at $72^{\circ} \mathrm{C}$. AP-PCR products were separated by electrophoresis in denaturing (4.0\% acrylamide and 50\% urea) sequencing gel and visualised by silver staining method (Singh and Roy, 1999). Each experiment was repeated three to four times.

\section{Cloning and sequencing of AP-PCR amplified product}

AP-PCR product of $475 \mathrm{bp}$ amplified by OPA14 (5'TCTGTGCTGG- $3^{\prime}$ ) primer was cloned and sequenced for further characterisation. This band in the AP-PCR fingerprint of uninvolved kidney DNA was excised from the gel, and DNA was eluted. The eluted DNA was reamplified with the same AP-PCR primer as before and using the same concentrations of reaction mixture constituents and PCR cycle conditions as described above. The PCR product was analysed on agarose gel to confirm its size and purity. The reamplified $475 \mathrm{bp}$ DNA was cloned using TA cloning kit (Invitrogen, Carlsbad, CA, USA). The presence of an appropriate insert size was determined by restriction analysis of recombinant plasmid DNA, and it was further confirmed by hybridising it with pure $475 \mathrm{bp} \mathrm{AP-PCR}$ amplified DNA as a probe. The insert was sequenced with automated DNA sequencer through DNA sequencing Core Facility, Comprehensive Cancer Center, UAB. Sequences obtained from several clones were compared with known sequences in the GenBank database using the BLASTn and BLASTx program.

\section{Southern blot analysis}

Total DNA was digested individually with restriction enzymes (BamH1, Sma1) according to the manufacturer's instructions and fractioned by $0.85 \%$ agarose gel electrophoresis (AGE) in TAE buffer (Maniatis et al, 1982). The separated DNA was transferred to nylon membrane (Hybond $\mathrm{N}^{+}$, Amersham Pharmacia Biotech Ltd.) by Southern blotting. The membrane was probed with $\left[\alpha-{ }^{32} \mathrm{P}\right]$-dCTP random primed-labelled $475 \mathrm{bp}$ AP-PCR amplified fragment (cloned) as SKCG-1 probe and the signals were detected by autoradiography of X-ray films. The same membrane was stripped off the SKCG-1 probe and then rehybridised with $G A P D H$ probe.

\section{Northern blot analysis}

Total RNA (5, 10, 20 $\mu \mathrm{g})$ isolated from HEK 293 cells was fractionated by electrophoresis in $1.0 \%$ agarose gel containing formaldehyde and transferred to Hybond- $\mathrm{N}^{+}$(Amersham) by capillary blotting. Membranes were hybridised with non-radioactive PCR-generated-DIG-labelled SKCG-1 or GAPDH (glyceraldehydes-3-phosphate dehydrogenase) gene-specific probes and washed according to protocols provided by the manufacturer of DIG high prime DNA labelling and detection kit (Roche).

\section{Isolation and sequencing of full-length SKCG-1 cDNA}

The full-length SKCG-1 cDNA sequence was obtained by EST sequencing and Rapid Amplification of cDNA ends (RACE) method as described previously (Frohman, 1993). A $1.37 \mathrm{~kb}$ sequence of SKCG-1 gene was obtained by sequencing of an EST clone (GenBank accession number AA935177) showing 100\% similarity with $475 \mathrm{bp}$ sequence. The remaining $(0.720 \mathrm{~kb})$ sequence was obtained by $5^{\prime}$ and $3^{\prime}$ RACE. $5^{\prime}$ and $3^{\prime}$ RACE-PCRs were carried out with total RNA obtained from normal human kidney and using the GeneRacer kit (Invitrogen). PCR reactions were carried out as specified by the manufacturer of GeneRacer kit (Invitrogen). To obtain $5^{\prime}$-ends, initial PCR was performed with gene-specific primer ( $5^{\prime}$-GCTGCGCTGTGGGTATGTAAGATGTT$3^{\prime}$ ) followed by a nested PCR with nested primer (5'-GGATACA CAGGGACTGCTTTG-3') to increase the gene specificity of PCR product. Similarly, $3^{\prime}$-ends sequence was obtained by $3^{\prime}$ RACE using gene-specific primer (5'-GGAGGCACCACTTGGTAACA-3') and nested PCR with primers ( $5^{\prime}$-CCTGAGTGTCTCTGCCGTGT$\left.3^{\prime}\right)$. These GSPs were designed based on sequence data obtained from $1.37 \mathrm{~kb}$ EST sequence. Final full-length cDNA sequence of 
SKCG-1, thus obtained, was deposited to NCBI GenBank and the assigned accession number is $A Y 662656$.

\section{Bioinformatic analysis of SKCG-1}

The sequence homology search and ORF analysis was performed by using software available at NCBI Website (www.ncbi.nlm.nih. gov). The prediction of potential methylation sites (CpG sites) in the promoter region sequence of SKCG-1 was analysed by computer-based free software (www.ebi.ac.uk/servicestmp). The type of protein (soluble or membrane) and its cellular localisation was predicted by using SOSUI software system (http://sosui. proteome.bio.tuat.ac.jp). The detail structure of SKCG-1 peptide, location of transmembrane region in the peptide, its hydropathy profile and helical wheel diagram is available at the EMBL bioinformatic harvester website (http://harvester.embl.de/harvester/ Q51SC8).

\section{Reverse transcriptase-polymerase chain reaction (RT - PCR)}

The transcript level of SKCG-1 was measured by semiquantitative PCR. Oligo dT-primed first strand cDNA was synthesised from DnaseI-treated total RNA $(2 \mu \mathrm{g})$ using Superscript II reverse transcriptase (Invitrogen). The PCR amplification was performed in a Perkin-Elmer Cetus DNA thermal cycler programmed for first cycle of $94^{\circ} \mathrm{C}$ for $3 \mathrm{~min}$, followed by 35 cycles $1 \mathrm{~min}$ of denaturation at $94^{\circ} \mathrm{C}, 1 \mathrm{~min}$ of annealing at $59^{\circ} \mathrm{C}, 1 \mathrm{~min}$ of extension at $72^{\circ} \mathrm{C}$. The PCR was completed by a final extension cycle at $72^{\circ} \mathrm{C}$ for $10 \mathrm{~min}$. PCR reactions with GAPDH-specific primers were also performed as control. SKCG-1 gene-specific PCR fragment of $300 \mathrm{bp}$ was amplified by forward ( $5^{\prime}$-GATAGGGAAGCCAAAGACAC- $3^{\prime}$ ) and reverse (5'-CCAGAGCAGGAGGATAATAAA-3 $\left.{ }^{\prime}\right)$ primers. Similarly, a $367 \mathrm{bp}$ fragment of housekeeping gene, GAPDH was amplified by forward ( $5^{\prime}$-GTCGCTGTTGAAGTCAGAGGA-3 ${ }^{\prime}$ ) and reverse $\left(5^{\prime}\right.$-TTCATGACAACTTTGGTATCG- $\left.3^{\prime}\right)$ primers. Samples were analysed by electrophoresis on $1.5 \%$ agarose gels.

\section{Tissue distribution of SKCG-1 gene expression}

The expression of SKCG-1 gene among the various tissues was analysed by PCR using tissue-specific cDNA panel (OriGene Technologies). The primers and the PCR conditions were the same as mentioned in the RT-PCR protocol. Reactions with $\beta$-actinspecific primers were used as a control according to the manufacturer's instructions.

\section{Chromosomal localisation of the SKCG-1 gene by fluorescence in situ hybridisation}

Chromosomal localisation of SKCG-1 gene was performed by FISH following the method of Stokke et al (1995). Human BAC clone genomic library was screened using original $475 \mathrm{bp}$ AP-PCR fragments as probe. Sequencing of the positive BAC clones using the primer designed from $475 \mathrm{bp}$ region revealed a clone with entire 475 bp sequences. Plasmid DNA was purified from this clone and was labelled with digoxigenin dUTP by nick translation. Labelled probe was combined with sheared human DNA and hybridised to normal metaphase chromosomes derived from phytohaemagglutinin-stimulated peripheral blood lymphocytes in a solution containing $50 \%$ formamide, $10 \%$ dextran sulfate and $2 \times$ SSC. Specific hybridisation signal were detected by incubating the hybridised slides in fluoresceinated anti-digoxigenin antibodies followed by counter staining with 4-6-diamidino2-phenylindole (DAPI) for one-colour experiments. Probe detection for two-colour experiments was accomplished by incubating the slides in fluoresceinated antidigoxigenin antibodies and Texas red followed by counterstaining with DAPI. Ideograms and estimates of distance of SKCG-1 gene locus from the centromere were calculated following the method as described by Francke (1994).

\section{SKCG-1 gene transcript silencing by siRNA}

Silencing of SKCG-1 was achieved by using the Dicer siRNA generation kit (Gene Therapy Systems, Inc., CA, USA) and following the manufacturer instructions. Briefly, double-stranded RNA (dsRNA) template was generated by T7 RNA polymerasemediated in vitro transcription from $500 \mathrm{bp}$ double-stranded target genomic region of $S K C G-1$. Double-stranded RNA was digested with the recombinant dicer enzyme to generate $22 \mathrm{bp}$ siRNA. Overnight grown $50-70 \%$ confluent adherent HEK293 cells were transfected with siRNA following the Lipofectamine method. To determine the extent of gene silencing, total RNA was isolated from siRNA transfected and untransfected (as control) HEK293 cells after $48 \mathrm{~h}$ of post-transfection. RT - PCR was performed using the total RNA and transcript level of SKCG-1 was determined following the method as described above in RT-PCR section. Cell growth was measured by counting the cells before isolating the RNA from these cells.

\section{RESULTS}

\section{Identification of mutation in human Wilms tumour samples}

To screen the mutations at genome-wide level, we compared the genomic fingerprint patterns of human Wilms tumours $v s$ matched nonmalignant (uninvolved) tissues by AP-PCR analysis. Out of 15 different random primers used, one primer (OPA14) revealed multifold decreased intensity of $475 \mathrm{bp}$ AP-PCR amplified DNA fragment in $38 \%$ ( 5 of 13 ) of the tumour samples as compared to uninvolved kidney tissues from the same individuals (Figure 1). Out of these five patients, one was male (5 years old CaucasianAmerican male, Figure 1, lane 5) and the remaining four were female (10 months old African-American female, Figure 1, lane 7; 5 years old Caucasian-American female, Figure 1, lane 9; 2 years old African-American female, Figure 1, lane 11; 4 years old CaucasianAmerican female, Figure 1, lane 22).

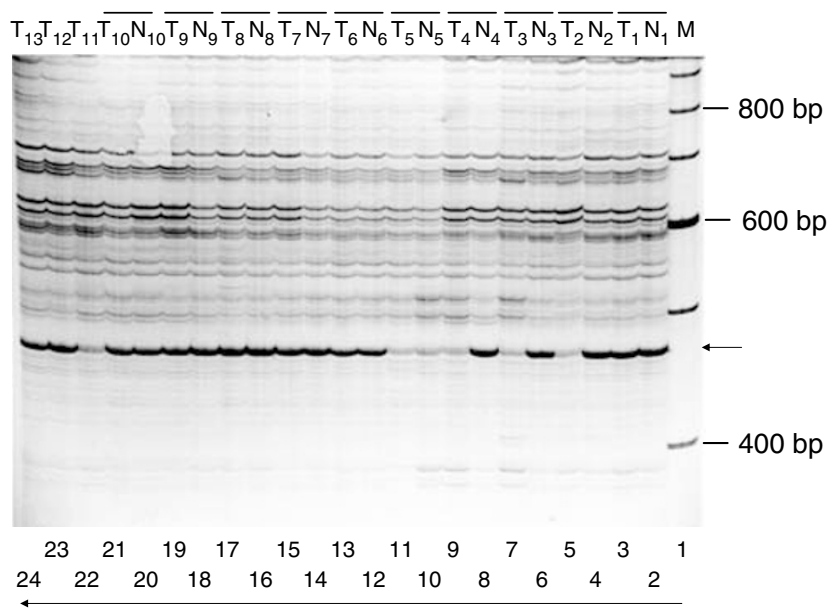

Figure I Representative AP-PCR fingerprints generated by primer OPAI4 (5'-TCTGTGCTGG-3') from Wilms tumours $\left(T_{1}-T_{13}\right)$ and corresponding uninvolved normal $\left(\mathrm{N}_{1}-\mathrm{N}_{13}\right)$ kidney tissue DNA. Arrow indicates the 475 bp AP-PCR amplified product with reduced intensity in 5 $\left(T_{2}, T_{3}, T_{4}, T_{5}\right.$, and $T_{11}$ of the Wilms tumour DNA samples as compared to the corresponding normal kidney tissue DNA from same patient. 
A

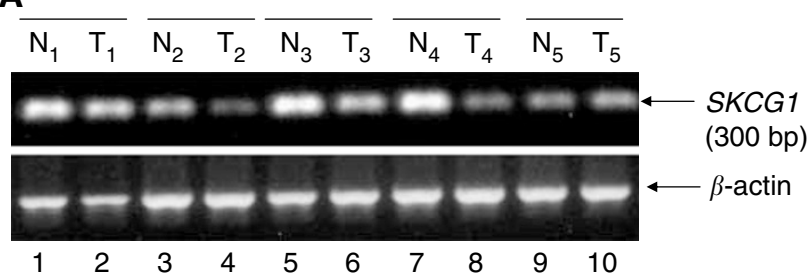

B

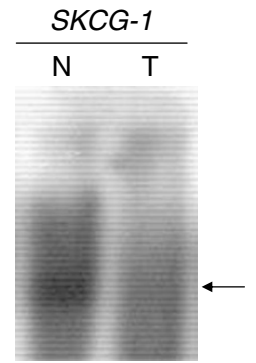

Bam HI digest

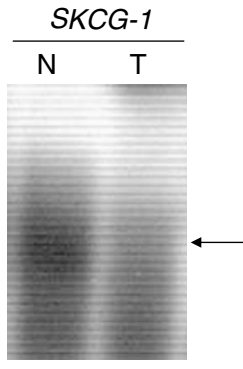

Sma I digest

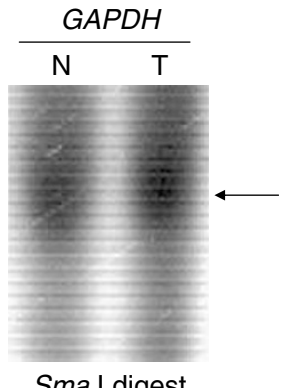

Sma I digest
Figure 2 Sequence characterised amplified region (SCAR) (A) and Southern blot (B) analysis showing genomic loss of SKCG-I in Wilms tumours $(T)$ and its presence in corresponding uninvolved normal $(N)$ kidney tissue. The primers used for SCAR amplification are mentioned in Materials and Methods section. Restriction enzymes, BamHI and Smal digested genomic DNA ( $10 \mu \mathrm{gs}$ ) from Wilms tumours ( $\mathrm{T}$ ) and corresponding uninvolved $(\mathrm{N})$ tissue was separated by electrophoresis, transferred to nylon membrane and hybridised with radioactive-labelled SKCG-I-or GAPDH-specific probe as mentioned in materials and methods. Arrow signs in figures indicate the hybridisation signal of SKCG-I in uninvolved normal tissue DNA and its loss in the tumour DNA sample. Control hybridisation with GAPDH (Glyceraldehyde-3-Phosphate Dehydrogenase) probe verified the equal amount of DNA loaded.

\section{Characterisation of mutation/deletion in $475 \mathrm{bp}$ genomic regions}

The cause for the multifold decreased intensity of $475 \mathrm{bp} \mathrm{AP-PCR}$ amplified locus in the tumour samples could be due to either mutation (s) at the primer-binding site(s) and/or due to deletion of this region in the genome of Wilms tumours.

Sequence Characterised Amplified Region (SCAR) analysis was performed to investigate whether the loss of $475 \mathrm{bp} \mathrm{AP-PCR}$ amplified region is a result of allelic loss. Using SKCG-1- specific primer as mentioned in RT-PCR section of materials and methods, we amplified genomic sequence of SKCG-1 from Wilms tumours and their corresponding uninvolved kidney tissue DNA. The combination of two SCAR primers generated a single PCR amplified fragment of expected size $(300 \mathrm{bp})$; however, the intensity of this fragment was reduced to almost half in tumours as compared to their corresponding uninvolved tissue samples (Figure 2A). The tumour samples showing reduced intensity in SCAR analysis were the same samples that revealed reduced intensity in $475 \mathrm{bp}$ AP-PCR amplified fragment (samples $\mathrm{T}_{2}, \mathrm{~T}_{3}, \mathrm{~T}_{4}$, $\mathrm{T}_{5}$ in Figures 1 and $2 \mathrm{~A}$ ). Thus, the result of SCAR analysis indicates loss of one allele of SKCG-1 in the tumours. Matching normal tissue samples were uninvolved surrounding tissue of tumours. One of the normal (N5) tissues DNA consistently produced a profile similar to the matching tumour in both AP-PCR (Figure 1, lane 10) and SCAR amplification (Figure 2A, lane 9). This could be explained by the presence of similar genetic changes in tumour as well as in the surrounding pathologically normal tissue (N5). Though these cells have undergone the genetic changes, however, they have still not been morphologically changed. Thus, some of the pathologically normal looking cells may not be genetically normal.

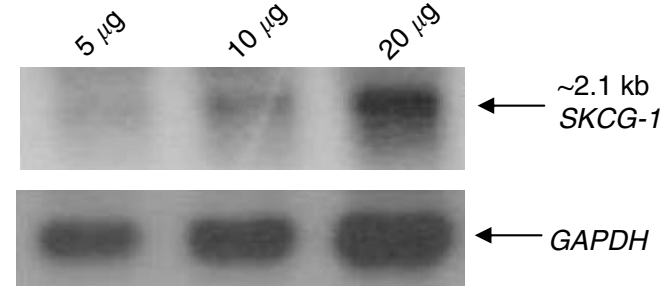

Figure 3 Northern blot analysis of SKCG-I. Total RNA isolated from immortalised embryonic kidney epithelial cells (HEK 293) was separated by electrophoresis, transferred to nylon membrane and hybridised with nonradioactive PCR generated-DIG-labelled SKCG-I gene-specific probe. Control hybridisation with GAPDH (Glyceraldehydes-3-phosphate dehydrogenase) probe verified the amount of RNA loaded.

We also performed Southern blot analysis with samples where enough DNA was available for this study. Presence of a hybridisation signal in uninvolved kidney tissue DNA and its absence in the corresponding tumour DNA was observed (Figure 2B). Thus, the result of Southern blot analysis confirmed the loss of SKCG-1 genomic region in Wilms tumour tissue.

In order to identify mutations, if any, in the SKCG-1 allele that is still present in the tumour, genomic sequence of SKCG-1 (from base +1 to +1692 ) was amplified from DNA of tumour as well as from matching normal tissue and sequenced. Comparison of sequence from normal tissue DNA with corresponding tumour tissue DNA did not reveal any mutation in the SKCG-1 allele in tumours.

\section{Determination of transcript size of SKCG-1 gene by Northern}

To determine the size of the SKCG-1 gene transcript, Northern analysis was performed with total RNA isolated from HEK293 cells derived from human embryonic kidney and using the PCRgenerated probe as described in material and methods. A single band corresponding to the SKCG-1 transcripts of $\sim 2.1 \mathrm{~kb}$ was observed (Figure 3).

\section{Isolation of full-length cDNA by RACE}

The AP-PCR amplified product of $475 \mathrm{bp}$ with primer OPA14 using DNA from uninvolved kidney tissue was gel eluted, reamplified, cloned and sequenced as described in material and methods. The final DNA sequence of $475 \mathrm{bp}$ AP-PCR fragment (Figure 4, from +1089 to +1564) obtained from five different clones was compared with the known gene/genomic sequence in the GenBank. BLAST analysis revealed that there was a complete match $(100 \%$ sequence similarity) of $475 \mathrm{bp}$ sequences with Homosapiens chromosome 11 working draft sequence segment (NT_033899.7) and genomic clone (accession number AP003027) from human chromosome $11 \mathrm{q}$ as well as with one of the human expressed sequence tag (EST) (accession number AA935177). Interestingly, this EST has been reported from the cDNA library made from human kidney tumour tissue. By sequencing of this EST clone, we obtained $1.37 \mathrm{~kb}$ of $S K C G-1$ (Figure 4, from +133 to +1503 ). Finally, $2.09 \mathrm{~kb}$ full-length cDNA sequence of SKCG-1 was obtained by performing $5^{\prime}$ and $3^{\prime}$ rapid amplification of cDNA ends (RACE) using the $1.37 \mathrm{~kb}$ sequence information. $5^{\prime}$ RACE added $0.133 \mathrm{~kb}$ (Figure 4, from +1 to +133 ) whereas the remaining $0.587 \mathrm{~kb}$ sequence (Figure 4, from +1503 to +2090 ) was obtained by $3^{\prime}$ RACE. The size of the $2.09 \mathrm{~kb}$ full-length transcript was very close to that of $2.1 \mathrm{~kb}$ as determined by northern. A comparison of the full-length cDNA sequence obtained by RACE with the genomic sequence revealed that this transcript is of single exon without any intervening intron sequence. Full-length cDNA sequence of SKCG-1 


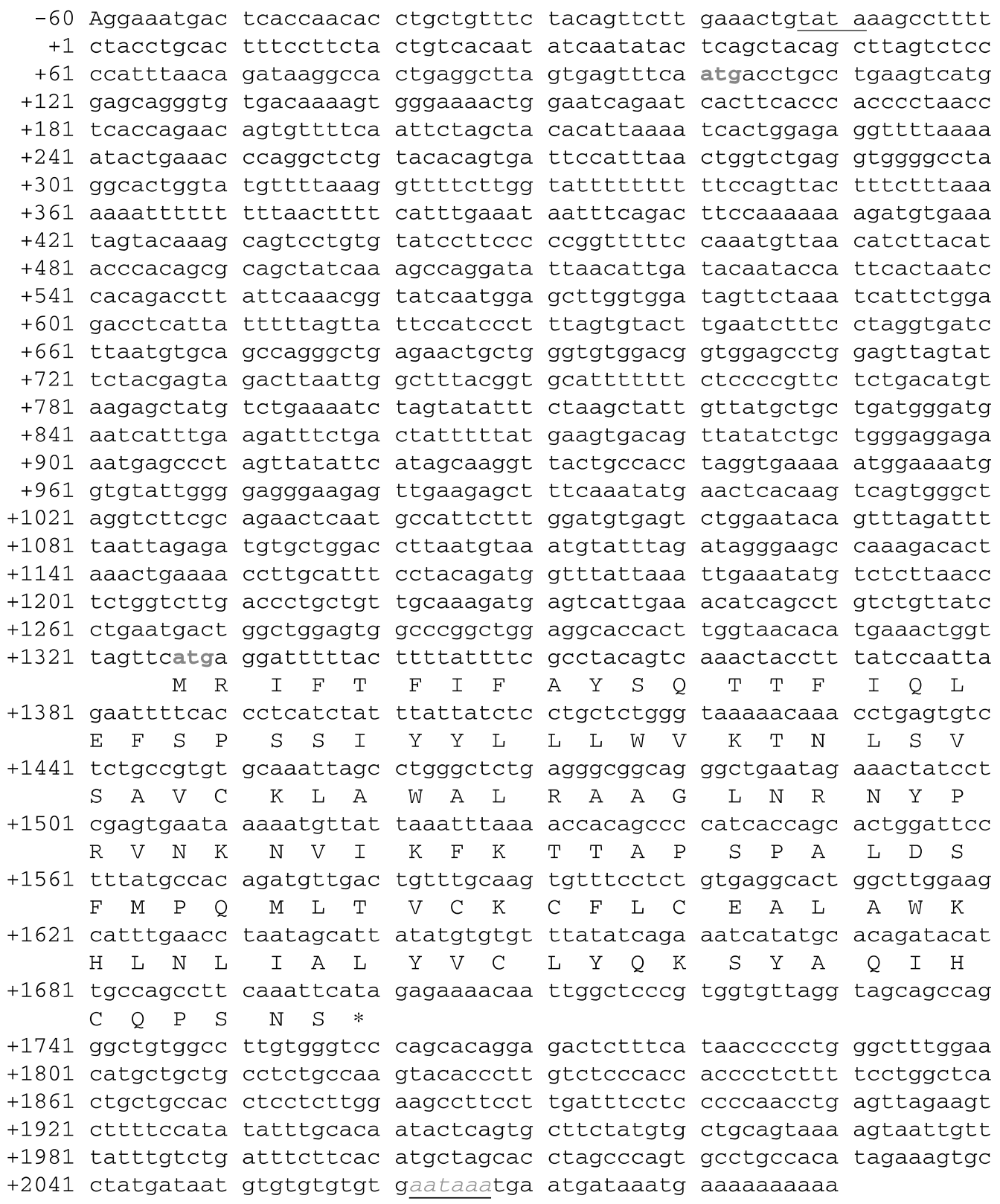

Figure 4 Full-length cDNA sequence and deduced amino-acid sequence of SKCG-I. The full-length SKCG-I cDNA encodes a predicted polypeptide of 124 amino acids. The polyadenylation signal is underlined. Sequence has been submitted to the NCBI database and the assigned GenBank accession number for SKCGI is AY662656.

has been submitted to the NCBI database and the assigned GenBank accession number for SKCG-1 is AY662656.

\section{Distribution of SKCG-1 gene expression in various human tissues}

To determine the distribution of SKCG-1 gene transcript in various human tissues, PCR amplification with SKCG-1 gene-specific primer was performed on a panel of cDNA from various human tissues (Origene Inc). SKCG-1 gene-specific amplification product of expected size $(300 \mathrm{bp})$ was abundantly present in brain, kidney, liver, testis, salivary gland, foetal brain, foetal liver, whereas relatively lower expression of this gene transcript was found in heart, small intestine, muscle, stomach, placenta, and prostate (Figure 5). No expression of this gene transcript was detected in spleen, colon, lung, adrenal gland, pancreas, uterus, skin, PBL, and bone marrow (Figure 5). An amplification product of higher size $(\sim 360 \mathrm{bp})$ in placenta and a lower size $(\sim 270 \mathrm{bp})$ in pancreas was observed (Figure 5).

\section{Expression of SKCG-1 gene transcript in Wilms and breast tumours, and their corresponding uninvolved tissue}

We examined the expression pattern of SKCG-1 gene transcripts by semi-quantitative RT-PCR analysis in Wilms $(N=4)$ and breast $(N=7)$ tumours and their corresponding uninvolved tissues. Expression of this gene was found to be either completely absent or hardly detectable in Wilms (Figure 6A) and breast (Figure 6B) tumours; however, its expression was readily detectable in their corresponding uninvolved tissues. These Wilms tumour samples 

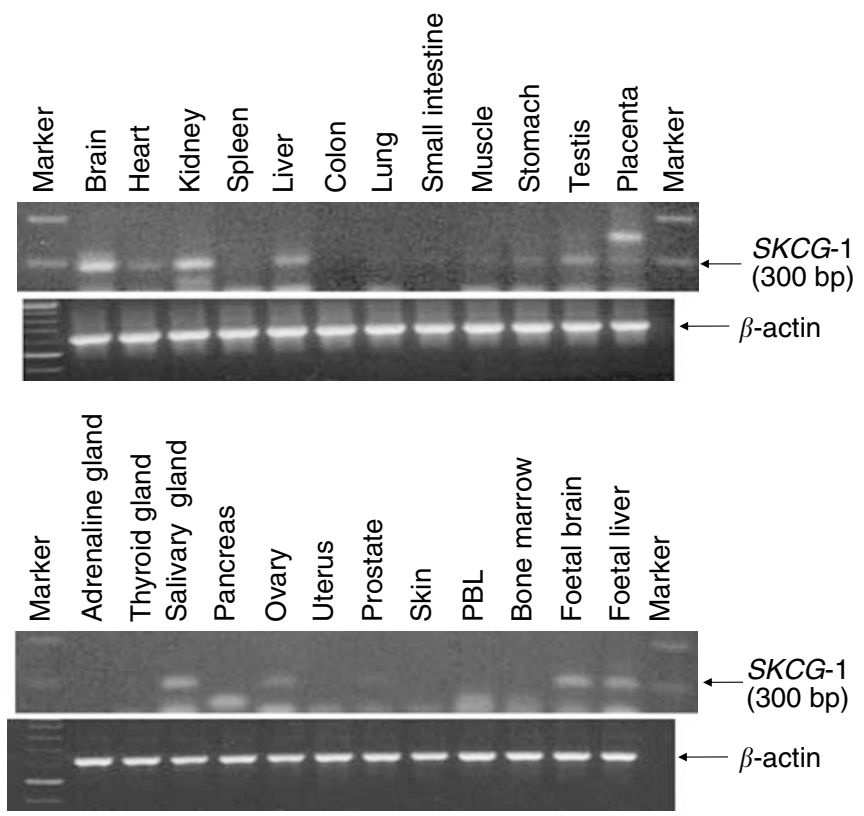

Figure 5 Distribution of SKCG-I gene expression in various human tissues. Expression of SKCG-I gene among the various tissues analysed by SKCG-I gene-specific PCR using tissue-specific cDNA panel (OriGene Technologies). Arrow indicates the expected size (300 bp) PCR product of SKCG-I from tissue-specific cDNA.

A

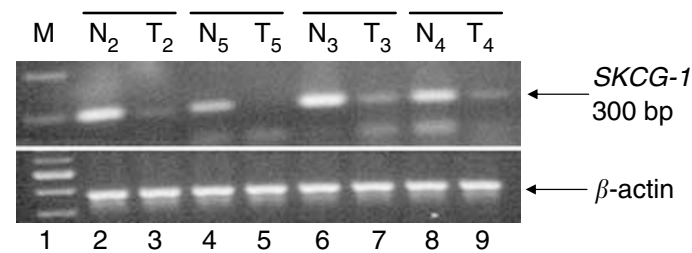

B

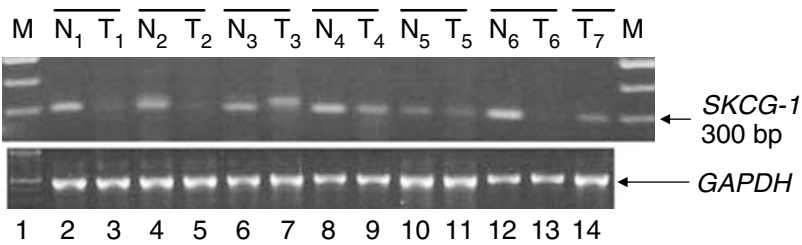

Figure 6 Semiquantitative RT-PCR analysis of SKCG-I transcript in human Wilms $(\mathbf{A})$, and breast $(\mathbf{B})$ tumours $(T)$ and corresponding uninvolved normal ( $\mathrm{N}$ ) kidney tissue. Amplification of $\beta$-actin or GAPDH was used as control for similar amount of RNA taken for RT reaction.

were the same samples that revealed loss of $475 \mathrm{bp}$ AP-PCR locus $\left(\mathrm{T}_{2}, \mathrm{~T}_{3}, \mathrm{~T}_{4}\right.$, and $\mathrm{T}_{5}$, Figures 1 and $\left.6 \mathrm{~A}\right)$. An amplification product of higher size $(320 \mathrm{bp})$ was observed in one of the breast tumour sample (Figure 6B, lane 7).

\section{Silencing of SKCG-1 by siRNA in HEK 293 cells}

To determine the extent of gene silencing by siRNA, the total RNA was isolated from HEK293 cells at 48-h post-transfection and semiquantitative RT-PCR was performed. The SKCG-1 gene transcript in siRNA-transfected cells was much lower as compared to the controls, indicating thereby the silencing of the gene (Figure 7A). To investigate whether the silencing of SKCG-1 gene has any effect on the cell growth, we performed cell counts before isolating the RNA. The cell counts data revealed a $40 \%$ increase in the growth of human embryonic kidney cells as a result of SKCG-1
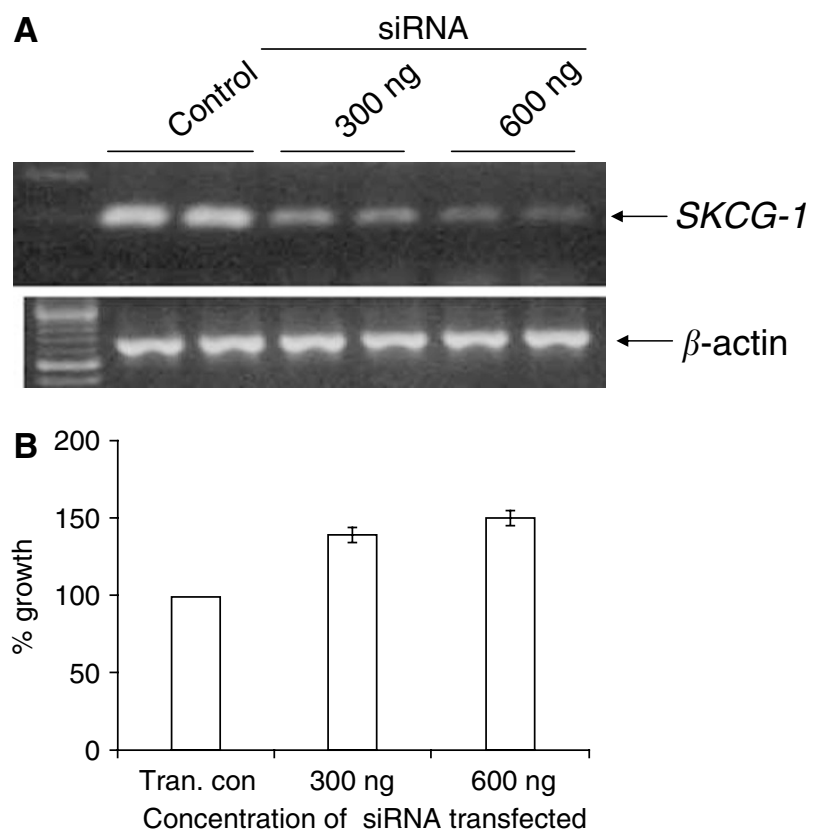

Figure 7 Effect of siRNA-mediated silencing of SKCG-I transcripts on the growth of human embryonic kidney epithelial cells. (A) SKCG-I genespecific siRNA oligos was transfected into HEK293 cells. Cells treated in parallel with transfection reagent without siRNA oligos served as control in transfection experiment. After $48 \mathrm{~h}$ of transfection, the total RNA was isolated, RT was performed, and transcript level was detected by PCR using SKCG-I specific primers. Amplification of $\beta$-actin was used as control for similar amount of RNA taken for RT reaction. (B) Histogram showing the siRNA concentrations transfected and its effect on the growth of HEK 293 cells.

gene silencing compared with the growth of control cells with constitutive expression of SKCG-1 (Figure 7B).

\section{Chromosomal localisation of SKCG-1 by fluorescence in situ hybridisation (FISH)}

Initial experiment of FISH with human BAC clone containing 475 bp region of $S K C G-1$ gene as a probe resulted in the specific hybridisation signal on the long arm of a group $\mathrm{C}$ chromosome that was believed to be chromosome 11 on the basis of size, morphology, and banding pattern. A second experiment with a biotin-labelled probe, which was specific for the centromere of chromosome 11, was cohybridised with SKCG-1 BAC clone. This experiment resulted in the specific labelling of the centromere in red and long arm in green of chromosome 11. Measurement of 10 specifically labelled chromosome 11 demonstrated that SKCG-1 BAC clone is located at a position, which is $73 \%$ of the distance from the centromere to the telomere of chromosome arm 11q, an area that corresponds to band 11q23.2 (Figure 8). A total of 80 metaphase cells were analysed with 75 exhibiting specific hybridisation signal.

\section{Structural analysis and coding potential of SKCG-1 gene}

Analysis of $2 \mathrm{~kb}$ upstream genomic sequence of SKCG-1 revealed a promoter region containing TATA box located at -13 bp upstream of transcription start site (underlined in Figure 4). Bioinformatic analysis using software program (www.ebi.ac.uk/servicestmp) revealed potential methylation sites ( $\mathrm{CpG}$ lots) located between -600 to $-900 \mathrm{bp}$ in the promoter region of SKCG-1. This region corresponds to nucleotide 106503-106803 of human genomic clone, NCBI accession number AP003027. Sequence analysis of 
$2.09 \mathrm{~kb}$ full-length cDNA revealed two translation start sites (ATG sequence) present at +101 and $+1327 \mathrm{bp}$ (represented by bold letters in Figure 4), and a polyadenylation signal sequence
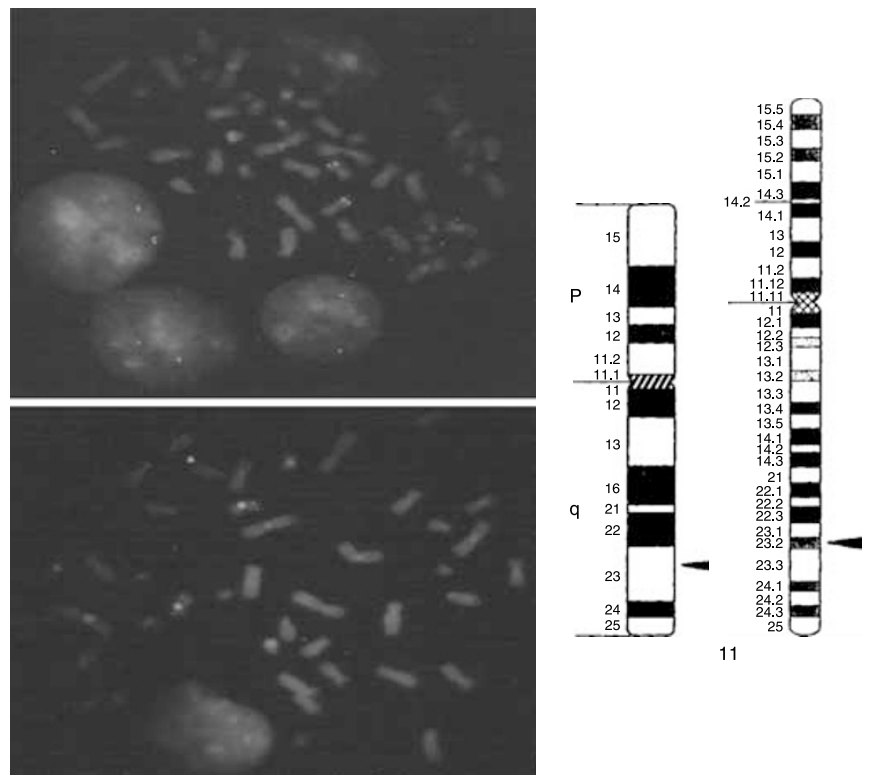

$\mathrm{FISH}$

Ideograms

Figure 8 Chromosome localisation of SKCG-I ascertained by fluorescence in situ hybridisation analysis. Left panel: Representative metaphase is shown exhibiting specific hybridisation signal (green fluorescent) on the long arm of chromosome II. A centromeric probe specific to the chromosome II was simultaneously hybridised (Red fluorescent). No signal was detected on any other chromosome using these probes. Right panel: Two ideograms illustrating the chromosomal position of SKCG-I at I l q23.2.
(AATAAA) present at $+2062 \mathrm{bp}$ (represented by italics in Figure 4). ORF analysis revealed that the cDNA sequence of this gene has an open reading frame encoding a protein of 124 amino acids in frame +1 (Figure 4). Domain analysis using NCBI conserve domain database revealed that this gene does not have any conserved functional domain. The EMBL bioinformatic harvester program Website has analysed the detail structure of SKCG-1 peptide. SOSUI software system has detected two transmembrane helices in the SKCG-1 peptide. The primary transmembrane helices is represented by transmembrane region (N-terminal, AAno.24)-SIYYLLLWVKTNLSVSAVCKLAW- (Cterminal, AAno.46) and the secondary transmembrane helices is represented by transmembrane region (N-terminal, AAno.86)VCKCFLCEALAWKHLNLIALYVC- (C-terminal, AAno.108) in $S K C G-1$ peptide. On the basis of the presence of these two transmembrane helices, the SKCG-1 has been predicted as a transmembrane protein. The predicted probability value of its localisation is $44.4 \%$ for extracellular including cell wall, $22.2 \%$ for cytoplasmic, $11.1 \%$ for golgi, $11.1 \%$ for nuclear, and $11.1 \%$ for endoplasmic reticulum. The detail structure of SKCG-1 peptide, location of transmembrane region and its hydropathy profile as well as helical wheel diagram is available at the EMBL bioinformatic harvester website (http://harvester.embl.de/harvester/ Q51SC8).

No significant homology (over full-length cDNA of SKCG-1) of this gene with any known gene in NCBI GenBank database was found. However, sequence similarity search using database of Celera Genomics revealed that SKCG-1 had $89 \%$ similarity over a 229 -nucleotide stretch and $85 \%$ similarity over a 62 -nucleotide stretch with a solute carrier family 14 (urea transporter) gene (Celera accession number NP_009094) reported from human kidney by Olives et al (1996) (Figure 9A). BLAST analysis revealed that a portion (from +497 to +522 ) of SKCG-1 gene has significant homology ( $96 \%$ identity) with several cDNA clones, for example, NCBI accession numbers BU542594, BU632939, BU543849, BQ013859, BM993263, BF002848), indicating thereby

A

16 agacagagtctcactctgtcacc-aggctgaagtgcagtggcgcaatctcagc

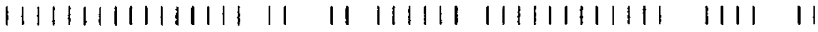

1895 agacagagtctcactccgttgcccaggctggagtgcagtggcgtgatcttggc

75 aa 76

11

1835 aa 1834

81 ggctcactgcaagctccgectccaggttcaagcgattctcctgcctcatcct

|l||||||||||||||||||||| |||||| || |||||||||||||| ||

1845 ggctcactgcaagctccacctcccgggttcacgccattctectgcctcagcct

141 agctgggactgeaggtgtgtgccaccacacccagctaattttt-gtagtttta 111111111111111 1111111 11 1111111111111 11111

1785 agctgggactacaggtgeccgecaccatgcctggctaatttttgtatttta

200 cggggtttcaccatgttggccaggatggtctcgatctcttgacctcgtgatcc 11111 1111111111111111111111111111111111111111 1725 tggggtctcaccatgttegccaggatggtctcgatctcctgacctcgtgatcc

260 ttggcetcccaaagtgctgggattacaggcatgagccgccatgcccggc 308 | $11111111111111111+11111111111111111111111111$

1665 teggectcccaaagtgetgggattacaggegtgagceaccgtgcecggc 161

B

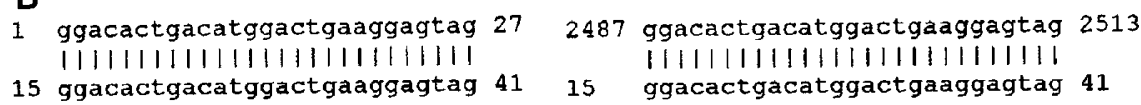

Figure 9 Sequence alignment at cDNA level of SKCG-I with a solute carrier family 14 (urea transporter) gene (A), and SOS2-like protein kinase mRNA (B). 
that this region represents a conserved sequence across these genes. Another potentially important region found was a stretch of 27 nucleotides starting from +1 ( $5^{\prime}$-end) that revealed $100 \%$ sequence identity with SOS2-like Protein Kinase mRNA, accession number AF 525402.1 (Figure 9B).

\section{DISCUSSION}

The most important finding of this study is the identification of an uncharacterised growth regulatory gene located at q23.2 region of chromosome 11. We observed genomic loss of this gene in $38 \%$ of the human Wilms tumour samples analysed. Several genes of pathogenic significance, for examples, $R C K, P L Z P, L P C, R C K$, in haematopoietic neoplasm have been mapped to $11 \mathrm{q} 23$ (Stilgenbauer et al, 1996). However, no gene/gene mutations have been identified at $11 \mathrm{q} 23.2$, and hence this is the first report of identification of a gene at this chromosomal region from Wilms tumour.

Our data indicate that the expression of $S K C G-1$ gene at transcript level is either completely absent or undetectable in Wilms and breast tumour samples. Interestingly, those Wilms tumour samples that had genomic loss of SKCG-1 also had reduced or loss of SKCG-1 transcript (Figures 1 and 6). This coincidence of loss at DNA level, and loss of gene expression at transcript level within a tumour indicates that presumably reduced/loss of this gene expression is a consequence of either complete loss (homozygous deletion) as observed in Southern blot analysis, or loss of one allele and/or silencing of remaining one allele by mutations/promoter methylation. Sequence analysis of the allele that is still present in tumours did not reveal any mutation. Thus more than $50 \%$ reduction in the expression in some samples could be due to loss of one allele by deletion and silencing of the remaining allele possibly by methylation of $S K C G-1$ promoter region. Presence of potential CpG lots in the $S K C G-1$ promoter region further supports the probability of $S K C G-1$ promoter region methylation as a causal factor for the silencing of expression of the allele that is still present in tumours. However, further study is needed to identify the exact cause for the loss of expression of SKCG-1 allele that is still present in tumour.

We did not find any association of the observed genetic alteration with either specific histopathology, grade and stage of tumour or age as well as race of the patients in the samples used in this study. However, it seems that this genetic alteration is more frequent in female genetic background than in male as four out of five samples showing genetic alteration and loss of gene expression were from female patients. This gender-associated genetic alteration in SKCG-1 remains to be validated by more number of samples.

Though we have not confirmed tumour suppressor activity of SKCG-1, however, the loss of expression of SKCG-1 in highly proliferative human Wilms and breast tumours suggest that it is a potential growth regulatory gene. A $40 \%$ increase in the cell growth as a result of siRNA-mediated silencing of this gene in human embryonic kidney epithelial cells further strengthens the fact that SKCG-1 controls the growth/proliferation of human kidney epithelial cells. The expression of $S K C G-1$ in various tissue including kidney and its loss in Wilms and breast tumours indicates that this gene is not kidney specific and it may have a role in other tissue too. Whether the SKCG-1 has similar growth regulatory function as observed in kidney tissue, and its loss of expression in their corresponding tumour tissue, remains to be investigated. The observed RT-PCR product of higher than the expected size in placenta and in one of the breast tissue samples, and lower than expected size in pancreas indicates that the spliced forms of SKCG-1 also exits.

The most striking feature of the SKCG-1 full-length cDNA sequence is the presence of very small open reading frames. The biggest open reading frame found in this gene was of 124 amino acids in frame +1 . The alternative use of CTG or ACG as the initiation codon for translation (Kozak, 1996) does not significantly lengthen any of the open reading frames. On the basis of the presence of polyadenylated RNA molecule, several genes have been ruled out being as pseudogenes (Bussemakers et al, 1999). As the $S K C G-1$ is expressed as polyadenylated RNA molecule with an ORF, and has function in controlling the cell growth as evident by siRNA silencing, the possibility of $S K C G-1$ gene as a pseudogene can be ruled out.

Thus, in the perspective of the function of this gene, there are two logical explanations: (i) either it encodes for a very small functional protein or, (ii) it acts as a riboregulator. The evidence in favour of the first explanation that it encodes for a small functional protein is that it has DNA sequence similarity with urea transporter gene and also with protein kinase. The prediction of SKCG-1 as a transmembrane protein based on the presence of two transmembrane helices in its peptide further strengthens the possibility of SKCG-1 as urea transporter gene in the membrane. However, further study is needed to ascertain whether it encodes any protein and its function as a transporter located in the membrane.

The other possibility of this gene being a riboregulator cannot be ruled out as well. In the last few years an emerging group of mRNA-like noncoding RNAs have been discovered whose function and mechanisms of action remain poorly understood (Erdmann et al, 2001). This group of RNA lacks a protein coding capacity, and most probably they exert their action mainly or exclusively at the RNA level. These RNA molecules that function as genetic regulators are also known as 'RNA riboregulator'. There are growing evidences which suggest that these riboregulators have important biological functions in development, differentiation, DNA damage, heat-shock responses, and tumorigenesis (Crespi et al, 1994; Velleca et al, 1994; Takeda et al, 1998). Several genes, for example, H19, His-1, Bic, that code for functional noncoding mRNAs have been found to play role in tumorigenesis (Askew and $\mathrm{Xu}, 1999)$. On the basis of our first report of identification and characterisation of SKCG-1 gene, we propose that SKCG-1 belongs to a distinct class of gene with potential function in kidney cell biology and in kidney tumorigenesis. In this report with several experiments we have provided the evidences for the role of SKCG-1 in growth control of malignant and normal kidney epithelial cells as well as its loss of expression in Wilms and breast tumours. Thus, our results support the potential value of SKCG-1 as new target for future clinical therapies to restore the normal cellular function of kidney and breast tissue.

\section{ACKNOWLEDGEMENTS}

This work was partly supported by the NIEHS Grant ES 10851 to DR, and financial support to KPS through NCI Cancer Prevention and Control Training Grant (CA 4788).

\section{REFERENCES}

Askew DS, Xu F (1999) New insights into the function of noncoding RNA and its potential role in disease pathogenesis. Histology Histopath 14(1): $235-241$
Bardeesy N, Falkoff D, Petruzzi MJ, Nowak N, Zabel B, Adam M, Aguiar MC, Grundy P, Shows T, Pelletier J (1994) Anaplastic Wilms tumour, a subtype displaying poor prognosis, harbors p53 gene mutations. Nat Genet 7(1): 91 - 97 
Bussemakers MG, Van Bokhoven A, Verhaegh GW, Smit FP, Karthaus HF, Schalken JA, Debruyne FM, Ru N, Isaacs WB (1999) DD3: A new prostate-specific gene highly overexpressed in prostate cancer. Cancer Res 59(23): $5975-5979$

Call KM, Glaser T, Ito CY, Buckler AJ, Pelletier J, Haber DA, Rose EA, Kral A, Yeger H, Lewis WH, Jones C, Houseman DE (1990) Isolation and characterisation of a zinc finger polypeptide gene at the human chromosome 11 Wilms tumour locus. Cell 60(3): 509-520

Crespi MD, Jurkevitch E, Poiret M, d'Aubenton-Carafa Y, Petrovics G, Kondorosi E, Kondorosi A (1994) Enod 40, a gene expressed during nodule organogenesis, codes for a non-translatable RNA involved in plant growth. EMBO J 13(21): 5099-5112

Erdmann VA, Barciszewska MZ, Szymanski M, Hochberg A, de Groot N, Barciszewski J (2001) The non-coding RNAs as riboregulators. Nucleic Acids Res 29(1): 189-193

Francke U (1994) Digitized and differentially shaded human chromosome ideograms for genomic applications. Cytogenet Cell Genet 65(3): $206-$ 218

Frohman MA (1993) Rapid amplification of complementary DNA ends for generation of full-length complementary DNAs: Thermal RACE. Meth Enzymol 218: 340 - 356

Gessler M, Poustka A, Cavenee W, Neve RL, Orkin SH, Bruns GA (1990) Homozygous deletion in Wilms tumours of a zinc-finger gene identified by chromosome jumping. Nature 343(6260): 774-778

Grundy PE, Telzerow PE, Breslow N, Moksness J, Huff V, Paterson MC (1994) Loss of heterozygosity for chromosomes $16 q$ and $1 p$ in Wilms' tumours predicts an adverse outcome. Cancer Res 54: $2331-2333$

Hing S, Lu YJ, Summersgill B, King-Underwood L, Nicholson J, Grundy P, Grundy R, Gessler M, Shipley J, Pritchard-Jones K (2001) Gain of 1q is associated with adverse outcome in favorable histology Wilms' tumours. Am J Pathol 158: $393-398$

Koufos A, Grundy P, Morgan K, Aleck KA, Hadro T, Lampkin BC, Kalbakji A, Cavenee WK (1989) Familial Wiedemann-Beckwith syndrome and a second Wilms tumour locus both map to 11p15.5. Am J Human Genet 44(5): $711-719$

Kozak M (1996) Interpreting cDNA sequences: some insights from studies on translation. Mammalian Genome 7(8): 563-574

Maniatis T, Fritsch EF, Sambrook J (1982) Molecular Cloning: A laboratory Manual. Cold Spring Harbor, New York
Mannens M, Slater RM, Heyting C, Bliek J, de Kraker J, Coad N, de PagterHolthuizen P, Pearson PL (1988) Molecular nature of genetic changes resulting in loss of heterozygosity of chromosome 11 in Wilms tumours. Human Genet 81(1): $41-48$

Matsunaga E (1981) Genetics of Wilms tumour. Hum Genet 57: $231-246$

Maw MA, Grundy PE, Millow LJ, Eccles MR, Dunn RS, Smith PJ, Feinberg AP, Law DJ, Paterson MC, Telzerow PE (1992) A third Wilms tumour locus on chromosome 16q. Cancer Res 52(11): 3094-3098

Olives B, Martial S, Mattei MG, Matassi G, Rouss RP, Cartron JP, Bailly P (1996) Molecular characterization of a new transporter in kidney. FEBS Lett 286(2-3): $156-160$

Radice P, Perotti D, De Benedetti V, Mondini P, Radice MT, Pilotti S, Luksch R, Fossati Bellani F, Pierotti MA (1995) Allelotyping in Wilms tumours identifies a putative third tumour suppressor gene on chromosome 11. Genomics 27(3): 497-501

Singh KP, Roy D (1999) Detection of mutation(s) or polymorphic loci in the genome of experimental animal and human cancer tissues by RAPD/AP PCR depend on DNA polymerase. Int J Oncol 14(4): 753-758

Singh KP, Roy D (2001) Identification of novel breast tumour-specific mutation(s) in the q11.2 region of chromosome 17 by RAPD/AP-PCR fingerprinting. Gene 269(1-2): 33-43

Stokke T, Collins C, Kuo WL, Kowbel D, Shadravan F, Tanner M, Lallioniemi A, Kallioniemi OP, Pinkel D, Deaven L (1995) A physical map of chromosome 20 established using fluorescence in situ hybridization and digital image analysis. Genomics 26(1): 134-137

Stilgenbauer S, Liebisch P, James MR, Schroder M, Schlegelberger B, Fischer K, Bentz M, Lichter P, Dohner H (1996) Molecular cytogenetic delineation of a novel critical genomic region in chromosome bands 11q22.3 - 23.1 in lymphoproliferative disorders. Proc Natl Acad Sci USA 93(21): $11837-11841$

Takeda K, Ichijo H, Fujii M, Mochida Y, Saitoh M, Nishitoh H, Sampath TK, Miyazono K (1998) Identification of a novel bone morphogenic protein-responsive gene that may function as a noncoding RNA. J Biol Chem 273(27): $17079-17085$

Velleca MA, Wallace MC, Merlie JP (1994) A novel synapse-associated noncoding RNA. Mol Cell Biol 14(11): 7095 - 7104

Volkher S, Alex J, Van der E, Jochemsen AG (2001) WT1 proteins: functions in growth and differentiations. Gene 273: $141-161$ 